\title{
Phase retrieval via propagation-based interferometry
}

\author{
L. Loetgering, ${ }^{*}$ H. Froese, and T. Wilhein \\ Institute for X-Optics, RheinAhrCampus Remagen, Joseph-Rovan-Allee 2, 53424 Remagen, Germany
}

M. Rose

Deutsches Elektronen-Synchrotron DESY, Hamburg, 22607, Germany

(Received 14 December 2016; published 17 March 2017)

\begin{abstract}
We present an extension of the Gerchberg-Saxton algorithm to allow for phase retrieval from interferometric data recorded at multiple sample-detector distances. A system of coupled waves is introduced where the information exchange can be controlled by use of relaxation parameters. Optimal parameters are investigated by numerical simulation and demonstrated to work with experimental data. We demonstrate that systematic errors such as pointing instabilities of the interfering waves involved and position uncertainties of the detector can be overcome by dimensional extension of the search space. Further it is shown that the proposed approach offers superior reconstruction quality as compared to conventional Gerchberg-Saxton type algorithms. We expect that the method described here will open up numerous possibilities for the correction of systematic errors in optical phase retrieval and lensless microscopy.
\end{abstract}

DOI: 10.1103/PhysRevA.95.033819

\section{INTRODUCTION}

In recent years, coherent diffraction imaging (CDI) [1] and related techniques such as ptychography $[2,3]$ have gained in importance due to their ability to recover both amplitude and phase information from intensity measurements of diffracted wave fields without refractive or diffractive optical elements. While in CDI a single diffraction pattern and a priori information are used to solve the phase problem, ptychography exploits transverse translation diversity by moving a sample through a stationary beam. Yet another approach to recovering the wave field from intensity diverse measurements is propagationbased phase retrieval, which was first investigated in electron microscopy $[4,5]$. Here the sample or the detector is moved coaxial to the beam propagation direction to yield intensities at various sample-detector distances. This approach is often combined with a Gerchberg-Saxton type reconstruction of the unknown wavefront [6-8]. However, while it was proven by Gerchberg and Saxton that the error in their iterative scheme monotonically decreases, to date there is still no guarantee for convergence. In addition, propagation-based phase retrieval is sensitive to misalignment of the experimental setup. It was shown recently that misalignment effects can be alleviated by means of a cross-correlation-based registration routine that can be embedded in the Gerchberg-Saxton iteration [9]. It was also shown that the same approach can be used to increase the numerical aperture and hence resolution of the optical imaging setup while no a priori knowledge about the specimen is needed.

In this paper, we present an extension of the aforementioned technique to the retrieval of both amplitude and phase information from interferometric data obtained at multiple sample-detector distances. The importance of this approach is threefold: (1) A system of relaxed coupled waves is proposed that imposes the interferometric constraint. The algorithm is tested by both simulation and experiment where it is shown that

\footnotetext{
*lars.loetgering@fulbrightmail.org
}

it outperforms the conventional Gerchberg-Saxton scheme. (2) The stability of the presented algorithm is examined and parameters that yield robust reconstructions are proposed. (3) It is shown that the method proposed here can compensate for systematic experimental errors such as pointing instabilities and position uncertainties of the detector by increasing the dimension of the search space of possible solutions to the phase retrieval problem.

\section{ALGORITHM}

The algorithm presented here aims at recovering wavefronts from a linear combination of unknown coherent waves from data diverse measurements. Similar to recent developments in ptychographic diffraction imaging [10], the method allows for robust phase retrieval in the case of beam instabilities by assuming the dimension of the search space of possible illuminations. Although the technique described here is not restricted to interferometric data, for illustration of the concept we consider here a Mach-Zehnder interferometer (MZI) as depicted in Fig. 1. A collimated beam traverses the interferometer with tilted mirrors. Blocking one arm at a time of the MZI yields the diffracted intensities $I_{1, z}=\left|\psi_{1, z}\right|^{2}$ and $I_{2, z}=\left|\psi_{2, z}\right|^{2}$. Coherent superposition of both exit-surface waves (ESWs) $\psi_{1, z}$ and $\psi_{2, z}$ yields the interferogram $I_{3, z}=$ $\left|\psi_{1, z}+\psi_{2, z}\right|^{2}$. In this notation, the index $z$ denotes the detector plane at distance $z$ from the object. In the object plane the index is omitted. Unlike in in-line holography, where an object wave is compared with a known reference wave, both $\psi_{1}$ and $\psi_{2}$ are unknown. The measured intensities $I_{1, z}, I_{2, z}$, and $I_{3, z}$ can be used as constraints employed iteratively in a system of coupled waves, i.e.,

$$
\left(\begin{array}{l}
\psi_{1}^{n+1} \\
\psi_{2}^{n+1} \\
\psi_{3}^{n+1}
\end{array}\right)=\left[\begin{array}{ccc}
\alpha & \alpha-1 & 1-\alpha \\
\beta-1 & \beta & 1-\beta \\
1-\gamma & 1-\gamma & \gamma
\end{array}\right]\left(\begin{array}{l}
\pi_{M}\left(\psi_{1}^{n}\right) \\
\pi_{M}\left(\psi_{2}^{n}\right) \\
\pi_{M}\left(\psi_{3}^{n}\right)
\end{array}\right),
$$

where $\alpha, \beta, \gamma \in[0,1]$ are relaxation parameters. Here $\pi_{M}$ is a modulus projector [11] incorporating the intensity constraint 


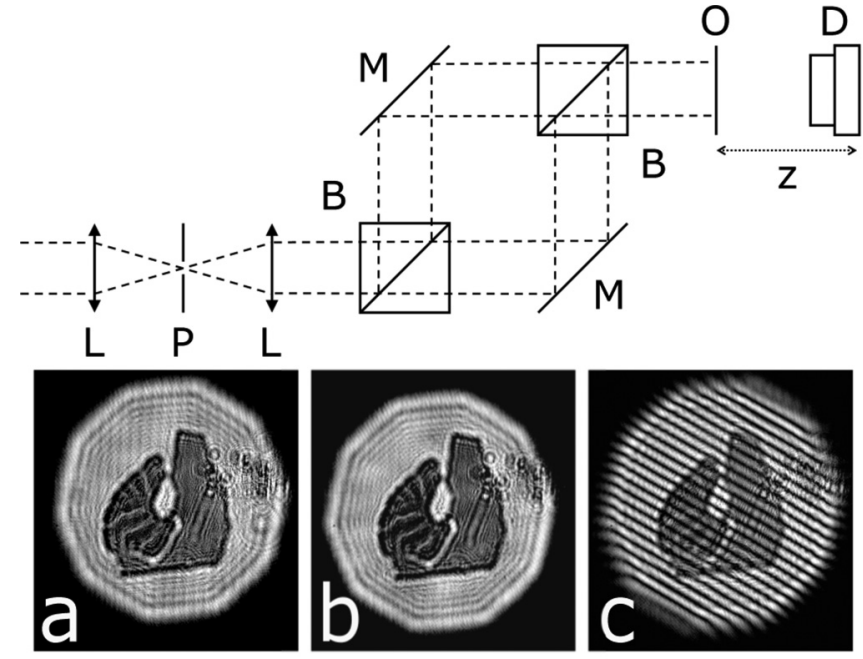

FIG. 1. The experimental setup (top) consists of a spatial filter (L-P-L) generating a collimated beam that traverses a Mach-Zehnder interferometer. The mirrors (M) of the Mach-Zehnder interferometer are aligned to produce beams with different pointing vectors that are used to produce interferometric data (bottom: (a) $\left|\psi_{1, z}\right|^{2}$, (b) $\left|\psi_{2, z}\right|^{2}$, (c) $\left|\psi_{1, z}+\psi_{2, z}\right|^{2}$ ) at multiple object (O)-detector (D) distances $(z)$.

of the $k$ th ESW at distance $z$ and iteration $n$ given by

$$
\pi_{M}\left(\psi_{k}^{n}\right)=\mathcal{D}_{z}^{-1}\left\{\sqrt{I_{k, z}} \frac{\mathcal{D}_{z} \psi_{k}^{n}}{\left|\mathcal{D}_{z} \psi_{k}^{n}\right|}\right\}
$$

where $k=1,2,3$ and $\mathcal{D}_{z}$ is an angular spectrum near-field propagator relating the wave fields in different planes [12], i.e., $\psi_{k, z}=\mathcal{D}_{z} \psi_{k}$. Equation (1) introduces a relaxation between the modulus and interferometric constraint for each respective ESW. The relaxation parameters allow us to control the relative strength of the modulus and interferometric constraints. The special case of $\alpha=\beta=\gamma=1$ corresponds to the GerchbergSaxton algorithm, where the waves are decoupled and Eq. (1) imposes only the modulus constraint. It is noted that Eq. (1) is prone to numerical instability when the constituting waves $\psi_{1}$ and $\psi_{2}$ exhibit pointing instability. The sensitivity of the interferogram to pointing instabilities is estimated to be on the order of $\lambda / \Delta x$, where $\Delta x$ is the spatial periodicity of the fringe pattern [13]. With fringe sizes on the order of typical detectors $(\Delta x \sim 10 \mathrm{~mm})$ and optical radiation $(\lambda \sim 500 \mathrm{~nm})$, it follows that the fringes undergo a phase shift of $2 \pi$ if the pointing instabilities of the waves $\psi_{1}$ and $\psi_{2}$ are on the order of $\mu \mathrm{rad}$. While this sensitivity is deliberately used in phase-shifting digital holographic interferometry [14], it leads to inconsistent fringes in propagation-based phase retrieval. In order to solve for $\psi_{k}(k=1,2,3)$ in the presence of mechanical instabilities, the following modified iterative algorithm is proposed: (1) Impose modulus constraint on constituent ESWs, i.e., $\psi_{1}^{n}=\pi_{M}\left(\psi_{1}^{n-1}\right)$ and $\psi_{2}^{n}=\pi_{M}\left(\psi_{2}^{n-1}\right)$. (2) Find an orthonormal basis $\xi_{1}^{n}, \xi_{2}^{n}, \ldots, \xi_{m}^{n}$ for the constituent waves $\psi_{1}^{n}, \psi_{2}^{n}$, where $m$ denotes the dimension of the search space of basis functions. (3) Project $\psi_{3}^{n-1}$ onto the vector space spanned by the orthonormal basis given by $\xi_{1}^{n}, \xi_{2}^{n}, \ldots, \xi_{m}^{n}$ and compute a relaxed update

$$
\begin{aligned}
\psi_{3}^{n} & =\gamma \psi_{3}^{n-1}+(1-\gamma) \pi_{\perp}\left(\psi_{3}^{n-1}\right) \\
& =\gamma \psi_{3}^{n-1}+(1-\gamma) \sum_{m}\left\langle\xi_{m}\left|\psi_{3}^{n-1}\right| \xi_{m},\right.
\end{aligned}
$$

where $\gamma \in[0,1]$. (4) Impose modulus constraint on interferometric ESWs, i.e., $\psi_{3}^{n+1}=\pi_{M}\left(\psi_{3}^{n}\right)$. (5) Find an orthonormal basis $\phi_{1}^{n}, \phi_{2}^{n}, \ldots, \phi_{m}^{n}$ for the set of updated interferometric ESWs $\psi_{3}^{n+1}$. (6) Update the constituent waves as

$$
\begin{aligned}
\psi_{1}^{n+1} & =\alpha \psi_{1}^{n}+(1-\alpha) \pi_{\perp}\left(\psi_{1}^{n}\right) \\
& =\alpha \psi_{1}^{n}+(1-\alpha) \sum_{m}\left\langle\phi_{m} \mid \psi_{1}^{n}\right\rangle \phi_{m}, \\
\psi_{2}^{n+1} & =\beta \psi_{2}^{n}+(1-\beta) \pi_{\perp}\left(\psi_{2}^{n}\right) \\
& =\beta \psi_{2}^{n}+(1-\beta) \sum_{m}\left\langle\phi_{m} \mid \psi_{2}^{n}\right\rangle \phi_{m},
\end{aligned}
$$

where $\alpha, \beta \in[0,1]$. The expansion coefficients in Eqs. (3), (4), and (5) denote inner products given by

$$
\langle\xi \mid \psi\rangle=\int \xi^{*}(x, y) \psi(x, y) d x d y,
$$

where $^{*}$ denotes complex conjugation and $\xi, \psi: \mathbb{R}^{2} \rightarrow \mathbb{C}$. The projector $\pi_{\perp}$, as defined by Eqs. (3), (4), and (5), is convex, which follows directly from the observation that the inner products in Eq. (6) are sesquilinear. In this regard the mathematical setting of the method proposed here is comparable to the shrink-wrap algorithm [15], where a convex support constraint, which is iteratively refined, is applied together with a nonconvex modulus constraint to invert diffraction data. Note that for the MZI setup in Fig. 1, the number of the basis function should ideally be chosen as $m=2$ to keep the computational complexity of each iteration low. However, as demonstrated in the simulations below, it can be advantageous to increase the search space to $m \geqslant 3$ in the presence of systematic experimental errors. It is noted that the steps (1) to (6) are executed sequentially and repeated iteratively for all $z$ distances in a randomized position order.

In the simulations and data analysis that follow, both orthonormal bases in steps 2 and 6 are computed by means of a truncated singular value decomposition [16]. Other methods as, for instance, the Gram-Schmidt method may be used as well [17]. Convergence of the algorithm at the $n$th iteration is monitored using the error metric

$$
\chi^{2}=\frac{\sum_{k} \sum_{z} \int\left(\sqrt{I_{k, z}}-\left|\mathcal{D}_{z} \psi_{k, z}^{n}\right|\right)^{2} d^{2} r}{\sum_{k} \sum_{z} \int I_{k, z} \mathrm{~d}^{2} r},
$$

where the summations range over all $z$ positions and ESWs.

\section{SIMULATION}

To test the algorithm proposed, numerical simulations assuming a detector with $512 \times 512$ pixels with a pixel size of $22 \mu \mathrm{m}$ and a wavelength of $488 \mathrm{~nm}$ were performed. To simulate beam vibrations, the tilts of the pointing vectors were randomized for each detector position such that the phase variation in the object plane ranges from 0 to $2 \pi$, causing quasirandom fringe movement between separate exposures. 


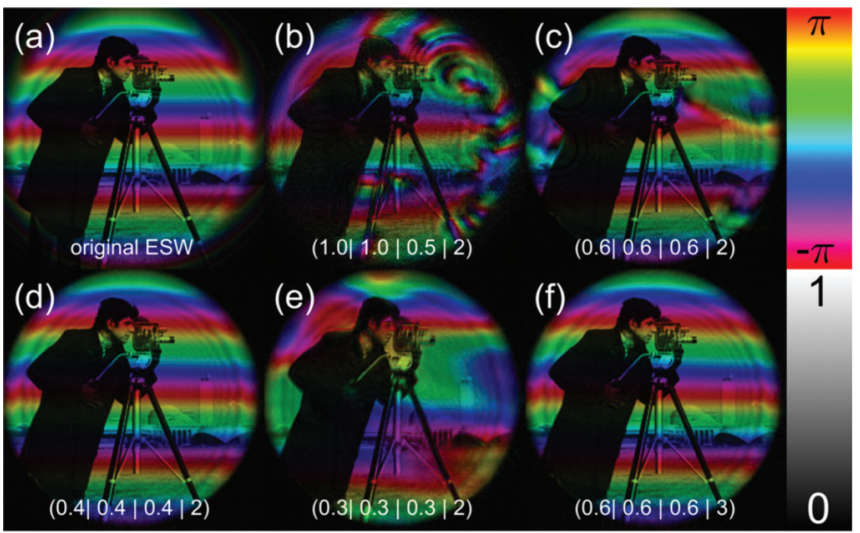

FIG. 2. Reconstruction results for simulated test data. Hue and brightness encode phase and magnitude, respectively, of the reconstructed wave field. The bottom of each subfigure indicates $(\alpha|\beta| \gamma \mid m)$, where $m$ indicates the number of basis functions used for reconstruction. Subfigure (a) shows one of the two original exit-surface waves. The coupled iterative scheme proposed, shown in panels (c)-(e), $\alpha=\beta=\gamma<1$, performs better than the uncoupled Gerchberg-Saxton type iteration shown in panel (b), $\alpha=\beta=1, \gamma=$ 0.5 . Comparing panels (c) and (f) indicates that increasing the number of basis functions can help to compensate systematic position errors.

The simulated ESWs as well as their superposition were propagated to eight distances ranging from 150 to $290 \mathrm{~mm}$, each distance of which was separated by $20 \mathrm{~mm}$. The position uncertainty was uniformly distributed in the interval $\pm 1 \mathrm{~mm}$. Poisson noise was simulated for each diffraction pattern with an average error of $\chi^{2}=5.1 \%$ as compared to the noise-free diffraction data. For a similar experimental configuration, successful wavefront sensing from multiple diffraction intensities was reported to require about 16 intensities when a GerchbergSaxton type reconstruction is deployed [18]. We chose half as many intensities to investigate whether the additional information provided by the interferograms can be used to successfully reconstruct the wave field from data that would fail in conventional Gerchberg-Saxton-type reconstructions. This is verified qualitatively and quantitatively in Figs. 2 and 3, which show reconstructions of the first ESWs and the $\chi^{2}$ error metric, respectively, for various combinations of coupling parameters $\alpha, \beta, \gamma$. For these reconstructions, an initial guess for the ESWs with both random amplitude and phase was assumed. In each iteration, the sequence of $z$ positions, from which data was updated, was randomized. This was observed to prevent the algorithm from getting stuck in premature convergence results. The phases of the final iterates where synchronized to facilitate comparison. With the number of basis functions chosen to be $m=2$, best reconstruction results were obtained for $\alpha=\beta=\gamma=0.4$, where the order of magnitude of $\chi^{2}$ monotonically decreased to the noise level after 30 iterations. Increasing the number of basis functions $(m=3)$ for other parameter choices exhibited improved reconstruction quality, as, for instance, seen by comparing Figs. 2(c) and 2(f), where $\alpha=\beta=\gamma=0.6$. For $m=5$, the reconstruction quality did not significantly improve further for any choice of parameters. Hence it is inferred that increasing the number of basis functions can help to compensate for systematic errors. In the absence of systematic errors, an

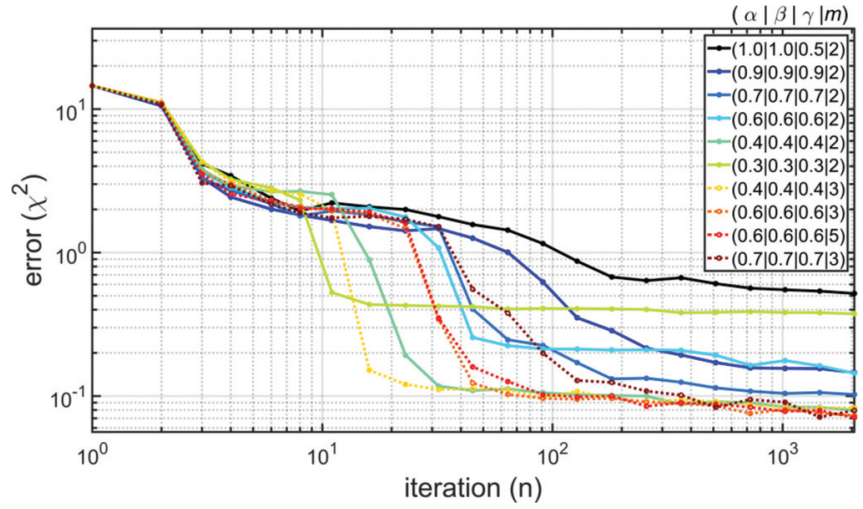

FIG. 3. Comparison of error metric $\left(\chi^{2}\right)$ for various parameters vs iteration $(n)$ for simulated data in the presence of systematic position errors and Poisson noise. Various coupling parameters and number of basis functions $(\alpha|\beta| \gamma \mid m)$ where tested for 2 (solid) and $\geqslant 3$ (dashed) basis functions. Best results for $m=2$ are obtained for $\alpha=\beta=\gamma=$ 0.4 . For $m \geqslant 3$ the reconstruction quality is less sensitive to the choice of coupling paramters.

increased number of modes did not yield any improvement in the reconstruction quality. For values $\alpha+\beta+\gamma>3$ (not shown here) the iterate diverged. It is noted that independent of parameter choice most iterates did not significantly improve the error metric after 200 iterations.

\section{EXPERIMENTAL RESULTS}

Experimental data were obtained using a collimated argonion laser $(\lambda=488 \mathrm{~nm}$, Spectra Physics model 2020). An aperture of $10 \mathrm{~mm}$ diameter was placed upstream of the MZI to prevent stray light from the sides of the beam splitters. A total of 60 diffraction patterns and interferograms were recorded on a 12-bit dynamic range CMOS detector (IDS UI-3370CP-M-GL) with $2048 \times 2048$ pixels and a pixel size of $5.5 \mu \mathrm{m}$ at 20 distances ranging from 40 to $135 \mathrm{~mm}$. The detector was moved in $\Delta z=5 \mathrm{~mm}$ steps. Each diffraction pattern and interferogram was corrected for background signal by subtracting dark frames. The diffraction patterns were aligned relative to each other using a gradient-based crosscorrelation registration routine. Each diffraction pattern $I_{z}$ was propagated into its adjacent detector position and compared with $I_{z+\Delta z}$. The relative transverse translation of the detector was computed by localizing the peak of the cross correlation of a template $T=\left(\partial_{x} \mathcal{D}_{\Delta z}+i \partial_{y} \mathcal{D}_{\Delta z}\right) \sqrt{I_{z}}$ and the reference $R=\left(\partial_{x}+i \partial_{y}\right) \sqrt{I_{z+\Delta z}}$. As shown in previous work [9], registration of longitudinally displaced diffraction patterns yields precise results if the displacement between adjacent diffraction patterns is smaller than the longitudinal speckle size. For slowly varying signals in the spatial domain, the gradient-based cross-correlation method was observed to yield more robust registrations than a standard cross-correlation method as the latter may yield compromised results in the presence of bias signal. The object under investigation is a sagittal histological section of a mouse cerebellum. The tissue was perfusion fixed in neutrally buffered $4 \%$ paraformaldehyde. After dissection, it was postfixed in the same fixative for $24 \mathrm{~h}$. It was then embedded in paraffin, and serial sections 


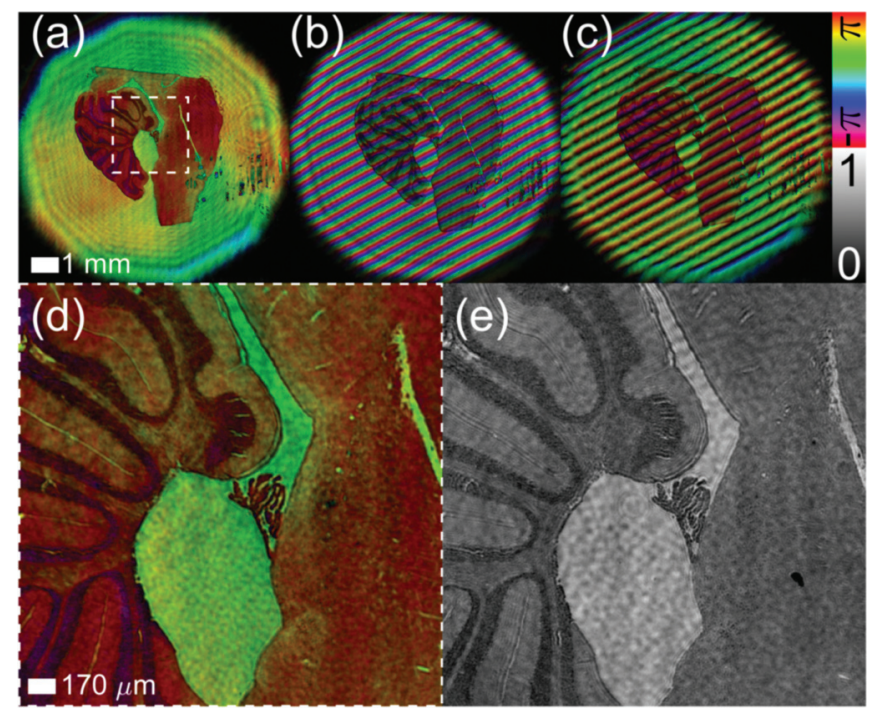

FIG. 4. Reconstruction of a stained mouse brain from 60 diffraction patterns and interferograms $(z=40, \ldots, 135 \mathrm{~mm})$. Hue represents phase shift, brightness relative modulus of the exit waves. Panels (a) and (b) show the constituting ESWs, and panel (c) is the interferometric ESW. The second ESW (b) exhibits a phase ramp that is robustly reconstructed due to the incorporation of the interferometric constraint. Panel (d) shows a magnified view of the white, dashed region in panel (a). (e) Optical micrograph of the same region as panel (d).

were cut at $10 \mu \mathrm{m}$. Sections were mounted on Superfrost Plus slides (VWR, Darmstadt, FRG), stained with hematoxylin and eosin, and finally covered using a 0.17 -mm-thick coverslip and DePeX (Serva, Heidelberg). The reconstructed ESWs using the algorithm described above $(\alpha=\beta=\gamma=0.4, m=2)$ are shown in Fig. 4, where transmission and phase shift are encoded by brightness and hue, respectively. The modulus of each ESW is normalized by its maximum to enhance contrast. The first ESW [Fig. 4(a)] shows an essentially plane phase profile with a small relative phase shift within the object region. The second ESW [Fig. 4(b)] exhibits a phase ramp that indicates a successful reconstruction of the relative phase as compared to the first ESW. The third ESW [Fig. 4(c)] depicts 1 out of 20 separately reconstructed interferograms. For comparison, Figs. 4(d) and 4(e) show the magnified complex-valued reconstruction of the white, dashed region in Fig. 4(a) and an optical micrograph, respectively, with similar numerical aperture (NA) as our lensless setup. The micrograph was recorded with a macro lens (70-mm F2.8 EX DG Macro) in a $4 f$ setup ( $\times 1$ magnification, 0.14 NA). Several reconstructions were carried out for varying choices of parameters. For $0.2<\alpha=\beta=\gamma<0.8$ ( $m=2,3,4)$ the reconstructions showed only minor differences, which might be due to the larger number of measured intensities as compared to the simulations carried out.

\section{DISCUSSION}

The numerical and experimental results presented here suggest that propagation-based phase retrieval from multiple detection planes can significantly benefit from the incorpora- tion of interferometric data. Consider, for example, the sets

$$
\begin{aligned}
& A=\left\{(x, y) \in \mathbb{R}^{2}:|2 x-y|^{2}=4\right\}, \\
& B=\left\{(x, y) \in \mathbb{R}^{2}:|2 y-x|^{2}=1\right\}, \\
& C=\left\{(x, y) \in \mathbb{R}^{2}:|x+y|^{2}=1\right\} .
\end{aligned}
$$

These sets may serve as a two-pixel model for a diffraction process, where $A, B$ describe diffraction patterns while $C$ describes interferometric data, where $x$ and $y$ are assumed to be real valued for simplicity. While $A \cap B=$ $\left\{\left(\frac{-5}{3}, \frac{-4}{3}\right),\left(\frac{5}{3}, \frac{4}{3}\right),(-1,0),(1,0)\right\}$, it is seen that $A \cap B \cap C=$ $\{(-1,0),(1,0)\}$. In this example, the interferometric information halves the solution set of the phase-retrieval problem. The additional constraints provided by the interferometric data impose only slight additional computational cost. For an $N \times N$ matrix, the Gerchberg-Saxton iteration is computed at the cost of the two-dimensional Fourier transform, i.e., $O\left(N^{2} \log _{2} N\right)$. The truncated singular value decomposition can be computed at cost $O\left(m N^{2}+m^{2} N\right)$, where $m$ is the number of basis functions used [16]. Assuming for most phase-retrieval applications $m \ll N$, it follows that an iteration of the algorithm presented here requires $O\left[N^{2}\left(m+\log _{2} N\right)\right]$ floating-point operations.

Stable parameters for the algorithm presented can be inferred from Eq. (1), which is a special case of the more general iteration given in Eqs. (3), (4), and (5). The characteristic polynomial of the matrix $M$ in Eq. (1) is given by

$$
\operatorname{det}(q I-M)=-(q-1)^{2}(q+2-\alpha-\beta-\gamma),
$$

where the roots of this polynomial in $q$ are eigenvalues of $M$. For the algorithm to iterate into a stable solution, the spectral radius of $M$ is required to be bounded by 1 , which yields the condition $1 \leqslant \alpha+\beta+\gamma \leqslant 3$. This condition is consistent with the observation that the algorithm presented diverges for $\alpha+\beta+\gamma>3$. In our simulations we observed that the relaxation parameters may initially be chosen close to unity for the algorithm to precondition the ESW estimates. If slow or no further progress is made, the relaxation parameters can be decreased to enable the interferometric data to improve the iterate. For $\alpha=\beta=\gamma$ each diffraction pattern and interferogram has an equal weight in the update rule given by Eqs. (3), (4), and (5). In this case, the above stability analysis suggests the choice $\alpha, \beta, \gamma \in[1 / 3,1]$. However, as demonstrated in Fig. 3 for $m=2$ the search performance of the algorithm varies significantly, while for $m>2$ the reconstruction quality is independent of the choice of relaxation parameters. We observed that the truncated SVD expansion can compensate for other experimental instabilities such as intensity and background fluctuations, since it only requires the data to be a linear combination of a set of shared basis functions. Similarly, a dimensional extension of the search space allows for the technique to apply to multibeam interference configurations.

\section{CONCLUSION}

In this paper, we have demonstrated interferometric phase retrieval from multiple sample-detector distances. It was shown numerically that the algorithmic approach reported here 
improves the Gerchberg-Saxton scheme by incorporation of interferometric constraints. Additionally, it was shown that an increase of the dimension of the search space of possible exitsurface waves can compensate for systematic experimental errors. The generality of this result may find applications in other fields of coherent imaging. Further, the experimental results suggest that the iterative scheme presented performs well in practice. As compared to other diffraction imaging modalities, neither a priori knowledge about the object under investigation nor a well-characterized phase plate or reference beam as required in coherent modulation imaging $[19,20]$ or digital holography, respectively, is needed. In comparison to (Fourier) ptychography $[21,22]$, no motion tracking of an optical stage nor knowledge of illumination wave fronts is necessary. Possible areas of applications may comprise digital holography with unknown references, wavefront sensing, and integrated interferometric configurations where stability of the setup cannot be maintained [23].

\section{ACKNOWLEDGMENTS}

L.L. would like to thank M. Odstrcil for useful discussions, M. Kschischo for help using the cluster at Rhein Ahr Campus Remagen, and K. Schilling for providing biological samples. This research was supported by the research initiative Rhineland-Pfalz. We gratefully acknowledge the support of NVIDIA Corporation with the donation of the Tesla K40 GPU used for this research.
[1] J. Miao, P. Charalambous, J. Kirz, and D. Sayre, Nature (London) 400, 342 (1999).

[2] P. Thibault, M. Dierolf, A. Menzel, O. Bunk, C. David, and F. Pfeiffer, Science 321, 379 (2008).

[3] H. M. L. Faulkner and J. M. Rodenburg, Phys. Rev. Lett. 93, 023903 (2004).

[4] R. W. Gerchberg and W. O. Saxton, Optik 35, 237 (1972).

[5] D. L. Misell, J. Phys. D: Appl. Phys. 6, L6 (1973).

[6] G. Pedrini, W. Osten, and Y. Zhang, Opt. Lett. 30, 833 (2005).

[7] J. Hagemann, A. L. Robisch, D. R. Luke, C. Homann, T. Hohage, P. Cloetens, H. Suhonen, and T. Salditt, Opt. Express 22, 11552 (2014).

[8] A. Ruhlandt, M. Krenkel, M. Bartels, and T. Salditt, Phys. Rev. A 89, 033847 (2014).

[9] L. Loetgering, R. Hammoud, L. Juschkin, and T. Wilhein, Europhys. Lett. 111, 64002 (2015).

[10] M. Odstrcil, P. Baksh, S. A. Boden, R. Card, J. E. Chad, J. G. Frey, and W. S. Brocklesby, Opt. Express 24, 8360 (2016).

[11] H. Stark, Image Recovery: Theory and Application (Academic Press, San Diego, CA, 1987), pp. 1-26.

[12] J. Goodman, Introduction to Fourier Optics, 3rd ed. (Roberts and Company Publishers, Englewood, CO, 2004).

[13] C. Cohen-Tannoudji, B. Diu, and F. Laloë, Quantum Mechanics, 1st ed. (Wiley, New York, 1977), Vol. 1, pp. 55-56.
[14] U. Schnars, C. Falldorf, J. Watson, and W. Jüptner, in Digital Holography (Springer-Verlag, Berlin, 2005), pp. 35-36.

[15] S. Marchesini, H. He, H. N. Chapman, S. P. Hau-Riege, A. Noy, M. R. Howells, U. Weierstall, and J. C. H. Spence, Phys. Rev. B 68, 140101 (2003).

[16] N. Halko, P. G. Martinsson, Y. Shkolnisky, and M. Tygert, SIAM J. Sci. Comput. 33, 2580 (2011).

[17] W. H. Press, S. A. Teukolsky, W. T. Vetterling, and B. P. Flannery, Numerical Recipes: The Art of Scientific Computing, 3rd ed. (Cambridge University Press, Cambridge, UK, 2007), p. 74.

[18] A. Anand, G. Pedrini, W. Osten, and P. Almoro, Opt. Lett. 32, 1584 (2007).

[19] F. Zhang, G. Pedrini, and W. Osten, Phys. Rev. A 75, 043805 (2007).

[20] F. Zhang, B. Chen, G. R. Morrison, J. Vila-Comamala, M. Guizar-Sicairos, and I. K. Robinson, Nat. Commun. 7, 13367 (2016).

[21] J. M. Rodenburg, A. C. Hurst, A. G. Cullis, B. R. Dobson, F. Pfeiffer, O. Bunk, C. David, K. Jefimovs, and I. Johnson, Phys. Rev. Lett. 98, 034801 (2007).

[22] G. Zheng, R. Horstmeyer, and C. Yang, Nat. Photon. 7, 739 (2013).

[23] C. Gorecki, F. Chollet, E. Bonnotte, and H. Kawakatsu, Opt. Lett. 22, 1784 (1997). 